\title{
Axiomatic characterization of the mean function on trees
}

\author{
F.R. McMorris \\ Department of Applied Mathematics, Illinois Institute of Technology \\ Chicago, IL 60616 USA \\ and \\ Department of Mathematics, University of Louisville \\ Louisville, KY 40292 USA \\ e-mail: mcmorris@iit.edu \\ Henry Martyn Mulder \\ Econometrisch Instituut, Erasmus Universiteit \\ P.O. Box 1738, 3000 DR Rotterdam, The Netherlands \\ e-mail: hmmulder@ese.eur.nl

\section{O. Ortega} \\ Department of Mathematics, Harold Washington College \\ Chicago, IL 60601 USA \\ e-mail: oortega@ccc.edu
}

\section{January 2010}

\section{Econometric Institute Report EI 2010-07}

This Technical Report is a Preprint, and it is not a formal publication in any sense. It will be submitted elsewhere.

\begin{abstract}
A mean of a sequence $\pi=\left(x_{1}, x_{2}, \ldots, x_{k}\right)$ of elements of a finite metric space $(X, d)$ is an element $x$ for which $\sum_{i=1}^{k} d^{2}\left(x, x_{i}\right)$ is minimum. The function Mean whose domain is the set of all finite sequences on $X$ and is defined by $\operatorname{Mean}(\pi)=\{x \mid x$ is a mean of $\pi\}$ is called the mean function on $X$. In this note, the mean function on finite trees is characterized axiomatically.
\end{abstract}

Keywords: Location function, mean function, median function, consensus function, tree 


\section{Introduction}

Let $(X, d)$ be a finite metric space and set $X^{*}=\bigcup_{k \geq 1} X^{k}$, where $X^{k}$ is the $k$-fold Cartesian product of $X$. Elements of $X^{*}$ are called profiles and denoted by $\pi=$ $\left(x_{1}, x_{2}, \ldots, x_{k}\right)$ with length $|\pi|=k$. A typical problem in location theory and consensus theory is the following: Given a collection of $k$ users (voters, customers, clients, etc.) with each user having a preferred location point in $X$, find a set of elements of $X$ that jointly satisfy the users with respect to some well-defined criteria. Modelling this situation requires, at the minimum, the notion of a location function on $X$, which is a function $L: X^{*} \longrightarrow 2^{X} \backslash\{\emptyset\}$, where $2^{X}$ denotes the set of all subsets of $X$. Because locating points "closest" to a given profile is a natural criterion, three straightforward examples of location functions are:

(a) The center function Cen with

$$
\operatorname{Cen}(\pi)=\{x \in X: e(x, \pi) \text { is minimum }\}
$$

where $e(x, \pi)=\max \left\{d\left(x, x_{1}\right), d\left(x, x_{2}\right), \ldots, d\left(x, x_{k}\right)\right\}$ is the eccentricity of $\pi$.

(b) The median function Med with

$$
\operatorname{Med}(\pi)=\left\{x \in X: S_{\pi}(x) \text { is minimum }\right\}
$$

where $S_{\pi}(x)=\sum_{i=1}^{k} d\left(x, x_{1}\right)$ is the status of $\pi$.

(c) The mean function Mean with

$$
\operatorname{Mean}(\pi)=\left\{x \in X: S S_{\pi}(x) \text { is minimum }\right\}
$$

where $S S_{\pi}(x)=\sum_{i=1}^{k} d^{2}\left(x, x_{1}\right)$ is the square status if $\pi$.

Location functions can be viewed as a special instance of consensus functions. A consensus function is a model to describe a rational process to obtain consensus among a group of agents or clients. The input of the function consists of certain information about the agents, and the output concerns the issue, about which consensus should be reached. The rationality of the process is guaranteed by the fact that the consensus function satisfies certain "rational" rules or "consensus axioms". A typical question in consensus theory is: which set of axioms characterizes a given consensus function. The theory of consensus is widely studied and used, e.g. in Economics and Social Choice Theory, see [1, 2], and in Biomathematics, see [6]. Holzman [8] was the first to study location functions from the axiomatic perspective.

We are concerned with location on finite connected graphs, so let $G=(V, E)$ be such a graph, and let $d$ be the usual distance function on $G$, where $d(x, y)$ is the length of a shortest path between $x$ and $y$. Clearly $(V, d)$ is a finite metric space. 
Notice that a profile on a graph $G$ is simply a sequence of vertices where repetitions are allowed. Among the location functions given above, the median function has been axiomatically characterized in several contexts, see e.g. [8, 3, 4, 10, 9]. In this note we consider the case when the graph is a tree $T=(V, E)$, i.e. a connected graph without cycles. McMorris, Roberts, and Wang [4] gave an axiomatic characterization for the center function on finite trees, see also [13], but not much is known for more general finite graphs. With respect to the mean function, Holzman [8] has given the first characterization of this function for trees in the "continuous case". Informally, a tree $T$ has an infinite number of elements in the continuous case, its edges are considered to be rectifiable curves, with $\pi$ and $L(\pi)$ allowed to be located anywhere on edges. In this context Vohra [14] has given an axiomatic characterization for the median function, and also a new characterization for the mean function using a set of axioms different from those used by Holzman. See Foster and Vohra [7] for related results.

Because the optimization criterion for the mean function is just as natural as that for the median, it is surprising that not much is known about the mean function on finite metric spaces from the axiomatic point of view. Biagi [5] finds a set of properties necessarily satisfied by the mean function on finite trees but they are not sufficient to provide a complete axiomatic characterization for the mean function. We will discuss this approach below as we move toward the goal of this paper, which is to give an axiomatic characterization of the mean function on finite trees.

\section{Axioms and Preparatory Results}

From now on $T=(V, E)$ will be used to represent a finite tree. If $\pi$ is a profile on $T$, then from [5] we know that $\operatorname{Mean}(\pi)$ is a $K_{1}$ or $K_{2}$ (one vertex, or two adjacent vertices).

The following axioms could reasonably be expected to be satisfied by a well-behaved location function. Indeed in [5] it is noted that the location function Mean satisfies these properties on a finite tree.

Let $L$ be a location function on $T=(V, E)$.

Faithfulness $(\mathbf{F}): L((x))=\{x\}$, for all $x \in V$.

Middleness (Mid) : Let $x, y \in V$. If $d(x, y)$ is even, then $L((x, y))=K_{1}$ where $d\left(x, K_{1}\right)=d\left(y, K_{1}\right)=d(x, y) / 2$. If $d(x, y)$ is odd, then $L((x, y))=K_{2}$ where $d\left(x, K_{2}\right)=d\left(y, K_{2}\right)$.

Consistency (C) : If $\pi_{1}=\left(x_{1}, x_{2}, \ldots, x_{k}\right)$ and $\pi_{2}=\left(y_{1}, y_{2}, \ldots, y_{s}\right)$ are profiles with $L\left(\pi_{1}\right) \cap L\left(\pi_{2}\right) \neq \emptyset$, then $L(\pi)=L\left(\pi_{1}\right) \cap L\left(\pi_{2}\right)$ with $\pi=\left(x_{1}, x_{2}, \ldots, x_{k}, y_{1}, y_{2}, \ldots, y_{s}\right)$.

Anonymity (A) : Let $\pi=\left(x_{1}, x_{2}, \ldots, x_{k}\right)$ be a profile on $T$ and let $\sigma$ be any permutation of $\{1,2, \ldots, k\}$, then $L(\pi)=L\left(\pi^{\sigma}\right)$, where $\pi^{\sigma}=\left(x_{\sigma(1)}, x_{\sigma(2)}, \ldots, x_{\sigma(p)}\right)$.

These four axioms are not independent: Faithfulness follows from Consistency and Middleness. 
Lemma 1 Let $L$ be a location function on $T$. If $L$ satisfies axioms (Mid) and $(C)$, then $L$ satisfies axiom $(F)$.

Proof. Let $z$ be a vertex of $T$ and let $\pi=(z, z)$. Since $d(z, z)=0$ is even, then by axiom (Mid) we have

$$
L(\pi)=L((z, z))=\{z\}
$$

On the other hand, $L((z))=L((z)) \cap L((z)) \neq \emptyset$, so axiom $(C)$ gives

$$
L((z)) \cap L((z))=L((z, z))=L(\pi)=\{z\} .
$$

and thus $\mathrm{L}((\mathrm{z}))=\{\mathrm{z}\}$.

In light of a similar result for the median function on trees [3], it was natural to conjecture that $(C),(M i d)$, and $(A)$ characterize Mean among all the location functions on trees. However, there are examples of location functions that satisfy axioms $(C),(M i d)$, and $(A)$ that are not equal to the mean function. For instance, let $\pi=\left(x_{1}, x_{2}, \ldots, x_{n}\right)$ be a profile on $T$ and define the cube status of a vertex $v$ with respect to $\pi$ as

$$
C S_{\pi}(v)=\sum_{i=1}^{k} d^{3}\left(v, x_{i}\right) .
$$

Now define the location function Cube by

$$
\text { Cube }(\pi)=\left\{v \in V \mid C S_{\pi}(v) \leq C S_{\pi}(x) \quad \forall x \in V\right\} .
$$

It was shown in [5] that $C$ ube satisfies $(C),(M i d)$, and $(A)$. To show that $M e a n(\pi) \neq$ $\operatorname{Cube}(\pi)$ consider the path $P\left(v_{1}, v_{10}\right)=v_{1} v_{2} \ldots v_{10}$ on ten vertices and the profile $\pi=\left(v_{1}, v_{2}, v_{10}\right)$ on $P\left(v_{1}, v_{10}\right)$. It is straightforward to verify that Mean $(\pi)=\left\{v_{4}\right\}$ and $\operatorname{Cube}(\pi)=\left\{v_{5}\right\}$.

Next, through a series of Lemmas, we establish properties of Mean and location functions that satisfy some of the previously listed axioms. This allows us to propose a new technical axiom that enables us to prove our main result in the next section.

The following lemma was proved in [5].

Lemma 2 Let $\pi=\left(x_{1}, x_{2}, \ldots, x_{n}\right)$ be a profile on $T$. If $u, v, w$ are distinct vertices such that $u w, w v \in E$, then

$$
S S_{\pi}(w)<\operatorname{Max}\left\{S S_{\pi}(u), S S_{\pi}(v)\right\} .
$$

Next we prove a lemma that can be viewed as an extension of Lemma 2 .

Lemma 3 Let $\pi=\left(x_{1}, x_{2}, \ldots, x_{n}\right)$ be a profile on $T$. If $m \in \operatorname{Mean}(\pi)$ and $P\left(m, y_{s}\right)=$ $m y_{1} y_{2} \ldots y_{s}$ is a path such that $y_{1} \notin \operatorname{Mean}(\pi)$, then

$$
S S_{\pi}(m)<S S_{\pi}\left(y_{1}\right)<S S_{\pi}\left(y_{2}\right)<\cdots<S S_{\pi}\left(y_{s}\right) .
$$


Proof. We use induction on $s$. The result is valid for $s=1$ because $y_{1} \notin \operatorname{Mean}(\pi)$ implies $S S_{\pi}(m)<S S_{\pi}\left(y_{1}\right)$. Assume the lemma is true for any path that contains at most $s$ vertices different than $m$ and let

$$
P\left(m, y_{s+1}\right)=m y_{1} y_{2} \ldots y_{s} y_{s+1}
$$

be a path such that $y_{1} \notin \operatorname{Mean}(\pi)$. The induction hypothesis implies

$$
S S_{\pi}\left(y_{s-1}\right)<S S_{\pi}\left(y_{s}\right) .
$$

Since $y_{s}$ is adjacent to both $y_{s-1}$ and $y_{s+1}$, then Lemma 2 yields

$$
S S_{\pi}\left(y_{s}\right)<\operatorname{Max}\left\{S S_{\pi}\left(y_{s-1}\right), S S_{\pi}\left(y_{s+1}\right)\right\} .
$$

Combining (3) and (4), we obtain $S S_{\pi}\left(y_{s}\right)<S S_{\pi}\left(y_{s+1}\right)$. This proves

$$
S S_{\pi}(m)<S S_{\pi}\left(y_{1}\right)<S S_{\pi}\left(y_{2}\right)<\cdots<S S_{\pi}\left(y_{s}\right)<S S_{\pi}\left(y_{s+1}\right),
$$

which completes the proof.

Since the mean of a profile on a tree is $K_{1}$ or $K_{2}$, the following is obvious.

Lemma 4 Let $\pi$ ) be a profile on $T$. Then Mean $(\pi)=\{a, b\}$ if and only if a is adjacent to $b$ and $S S_{\pi}(a)=S S_{\pi}(b)$.

The process of finding a vertex $x$ that belongs to the set Mean $(\pi)$ is a global property, which requires comparing $S S_{\pi}(x)$ against the square status of every vertex on $T$. If we know in advance that the mean of $\pi$ is a set with two elements, then Lemma 4 gives a method that avoids a lot of calculations. For any given vertex $x$, we first obtain the set of neighbors of $x$, and if we are lucky to find in this set a vertex $y$ satisfying the condition $S S_{\pi}(x)=S S_{\pi}(y)$, then we know that $\operatorname{Mean}(\pi)=\{x, y\}$.

We would like to have a similar local procedure that we can apply when $|\operatorname{Mean}(\pi)|=$ 1. Notice that if $u \in \operatorname{Mean}(\pi)$, then

$$
S S_{\pi}(u) \leq S S_{\pi}(z) \quad \forall z \in V .
$$

In particular

$$
S S_{\pi}(u) \leq S S_{\pi}(z)
$$

for any vertex $z$ adjacent to $u$. This suggests that in trying to discover if a vertex $x$ is in the set $\operatorname{Mean}(\pi)$, we could first verify that $S S_{\pi}(x)$ is less or equal than the square status of every neighbor of $x$. If that conditions holds, then $x$ is a good candidate to be in the mean of $\pi$. This argument motivates the following definition.

Definition 1 Let $\pi$ be a profile on $T$. A vertex $u$ satisfies the mean condition with respect to $\pi$ if

$$
S S_{\pi}(u) \leq S S_{\pi}(z)
$$

for any vertex $z$ adjacent to $u$. 
The following lemma shows that the elements of the set Mean $(\pi)$ are precisely the vertices that satisfy the mean condition with respect to $\pi$.

Lemma 5 Let $\pi$ be a profile on $T$. Then $u \in \operatorname{Mean}(\pi)$ if and only if $u$ satisfies the mean condition with respect to $\pi$.

Proof. It is clear that if $u \in$ Mean $(\pi)$, then $u$ satisfies the mean condition with respect to $\pi$. Conversely, let $u$ be a vertex satisfying the mean condition with respect to $\pi$ and assume $u \notin \operatorname{Mean}(\pi)$. Let $x \in \operatorname{Mean}(\pi)$ be a vertex such that $d(\operatorname{Mean}(\pi), u)=$ $d(x, u)$, and let

$$
P(x, u)=x v_{1} v_{2} \ldots v_{s} u
$$

be the path from $x$ to $u$. By Lemma 3 we obtain

$$
S S_{\pi}(x)<S S_{\pi}\left(v_{1}\right)<S S_{\pi}\left(v_{2}\right)<\cdots<S S_{\pi}\left(v_{s}\right)<S S_{\pi}(u) .
$$

We see that $v_{s}$ is adjacent to $u$ and $S S_{\pi}\left(v_{s}\right)<S S_{\pi}(u)$. However, this contradicts that $u$ satisfies the mean condition with respect to $\pi$. This shows that $u \in \operatorname{Mean}(\pi)$.

Let $\pi=\left(x_{1}, x_{2}, \ldots, x_{n}\right)$ be a profile on $T$, and let $z \in V$. Define the profile $\pi \diamond z$ as follows

$$
\pi \diamond z=\left(x_{1}, x_{2}, \ldots, x_{n}, z\right)
$$

Lemma 6 Let $\pi=\left(x_{1}, x_{2}, \ldots, x_{n}\right)$ be a profile on $T$. Let $L$ be a location function satisfying axioms $(F)$ and $(C)$. If $z \in L(\pi)$, then $L(\pi \diamond z)=\{z\}$.

Proof. Define the profile $\beta=(z)$, and notice that axioms $(F)$ and $(C)$ imply

$$
L(\pi \diamond z)=L(\pi) \cap L(\beta)=\{z\},
$$

and we are done.

The next lemma shows that the mean function satisfies also the converse of Lemma 6 .

Lemma 7 Let $\pi$ be a profile on T. If $y$ is a vertex such that Mean $(\pi \diamond y)=\{y\}$, then $y \in \operatorname{Mean}(\pi)$.

Proof. Let $z$ be a vertex adjacent to $y$, and observe that $M e a n(\pi \diamond y)=\{y\}$ implies

$$
S S_{\pi \diamond y}(y)<S S_{\pi \diamond y}(z)
$$

Note that

$$
\begin{aligned}
& S S_{\pi \diamond y}(y)=S S_{\pi}(y), \\
& S S_{\pi \diamond y}(z)=S S_{\pi}(z)+1,
\end{aligned}
$$


which means

$$
S S_{\pi}(y) \leq S S_{\pi}(z)
$$

Since this is true for any vertex $z$ adjacent to $y$, it follows that $y$ satisfies the mean condition with respect to $\pi$. By Lemma 5, we have $y \in \operatorname{Mean}(\pi)$.

In the search for mean vertices we might proceed as follows. We are at vertex $a$ and compare the square status of $a$ with that of an adjacent vertex $b$. If the square status of $b$ is smaller than that of $a$ we "improve" our position by moving to $b$. Now we compare the square status of $b$ with that of a new vertex $c$ adjacent to $b$. Thus we have to compare the square status of adjacent vertices but also of three consecutive vertices on a path of length 2 . Therefore, we introduce the following notation.

Let $\pi=\left(x_{1}, x_{2}, \ldots, x_{n}\right)$ be a profile on $T$ and assume $a b, b c \in E$. Denote by $\pi_{a b}$ the profile that contains the elements $x$ of $\pi$ (in the same order) that are closer to $a$ than to $b$, that is, satisfy the condition

$$
d(a, x)+1=d(b, x) .
$$

Notice that the vertex $x$ appears in $\pi_{a b}$ as many times as it appears in $\pi$.

Similarly, denote by $\pi_{a b c}$ the profile that contains the elements $x$ of $\pi$ that satisfy the following conditions

$$
\begin{aligned}
& d(b, x)+1=d(a, x), \\
& d(b, x)+1=d(c, x) .
\end{aligned}
$$

We use these profiles to define two quantities. The first could be considered as a surplus when comparing the square status of the adjacent vertices $a$ and $b$. The second one could be considered as an indifference count with respect to $a$ and $c$ by computing the distance sum of all the vertices having equal distance to $a$ and $c$. The use of these quantities will become clear in the following computations.

$$
\begin{aligned}
R_{\pi}(a, b) & =\sum_{x \in \pi_{b a}} d(b, x)-\sum_{x \in \pi_{a b}} d(b, x), \\
D_{\pi}(a, b, c) & =2 \sum_{x \in \pi_{a b c}} d(b, x) .
\end{aligned}
$$

By Anonymity we may write

$$
\pi=\pi_{a b} \pi_{b a} \quad \text { as well as } \quad \pi=\pi_{a b} \pi_{a b c} \pi_{c b} .
$$

The representation of $\pi$ as $\pi=\pi_{a b} \pi_{b a}$ leads to the following relationship between $R_{\pi}(a, b), S S_{\pi}(b)$, and $S S_{\pi}(a)$.

$$
S S_{\pi}(a)-S S_{\pi}(b)=2 R_{\pi}(a, b)+|\pi| .
$$


Using the identity $S S_{\pi}(a)-S S_{\pi}(b)=-\left[S S_{\pi}(b)-S S_{\pi}(a)\right]$ and (5) we get the following connection between $R_{\pi}(a, b)$ and $R_{\pi}(b, a)$ :

$$
R_{\pi}(a, b)=-R_{\pi}(b, a)-|\pi| .
$$

Similarly, from $\pi=\pi_{a b} \pi_{a b c} \pi_{c b}$ we obtain

$$
\begin{aligned}
& S S_{\pi}(a)-S S_{\pi}(b)=-2 \sum_{x \in \pi_{a b}} d(b, x)+2 \sum_{x \in \pi_{a b c}} d(b, x)+2 \sum_{x \in \pi_{c b}} d(b, x)+|\pi| \\
& S S_{\pi}(c)-S S_{\pi}(b)=2 \sum_{x \in \pi_{a b}} d(b, x)+2 \sum_{x \in \pi_{a b c}} d(b, x)-2 \sum_{x \in \pi_{c b}} d(b, x)+|\pi| .
\end{aligned}
$$

Next we prove two lemmas that we need later.

Lemma 8 Let $\pi=\left(x_{1}, x_{2}, \ldots, x_{n}\right)$ be a profile on $T$. If $a b, b c \in E$, then

$$
R_{\pi}(a, b)+R_{\pi}(c, b)=D_{\pi}(a, b, c) .
$$

Proof. Adding (7) and (8), we get

$$
\left[S S_{\pi}(a)-S S_{\pi}(b)\right]+\left[S S_{\pi}(c)-S S_{\pi}(b)\right]=4 \sum_{x \in \pi_{a b c}} d(b, x)+2|\pi| .
$$

From (5) we obtain

$$
\begin{gathered}
S S_{\pi}(a)-S S_{\pi}(b)=2 R_{\pi}(a, b)+|\pi| \\
S S_{\pi}(c)-S S_{\pi}(b)=2 R_{\pi}(c, b)+|\pi| .
\end{gathered}
$$

Notice also that

$$
4 \sum_{x \in \pi_{a b c}} d(b, x)=2 D_{\pi}(a, b, c) .
$$

When we substitute these identities in (9), we obtain

$$
R_{\pi}(a, b)+R_{\pi}(c, b)=D_{\pi}(a, b, c),
$$

which completes the proof.

Lemma 9 Let $\pi=\left(x_{1}, x_{2}, \ldots, x_{n}\right)$ be a profile on $T$. If $|\pi|$ is odd, then $\mid$ Mean $(\pi) \mid=1$.

Proof. Assume $\operatorname{Mean}(\pi)=\{a, b\}$, then $S S_{\pi}(b)=S S_{\pi}(a)$. Since $a$ is adjacent to $b$, it follows from (5) that

$$
S S_{\pi}(b)-S S_{\pi}(a)=2 R_{\pi}(b, a)+|\pi|=0 .
$$

This means that $|\pi|$ is even, and contradicts the assumption that $|\pi|$ is odd. Consequently, we have $|\operatorname{Mean}(\pi)|=1$.

Our next result will be used to define a special property for location functions. 
Lemma 10 Let $\pi$ be a profile on $T$. If $a b, b c \in E$ and $S S_{\pi}(a)<S S_{\pi}(b)<S S_{\pi}(c)$, then $R_{\pi}(b, a)+R_{\pi}(c, b)>D_{\pi}(a, b, c)$.

Proof. From (7) we obtain

$$
S S_{\pi}(b)-S S_{\pi}(a)=2 \sum_{x \in \pi_{a b}} d(b, x)-2 \sum_{x \in \pi_{a b c}} d(b, x)-2 \sum_{x \in \pi_{c b}} d(b, x)-|\pi| .
$$

Adding (10) and (8), we get

$$
\left[S S_{\pi}(b)-S S_{\pi}(a)\right]+\left[S S_{\pi}(c)-S S_{\pi}(b)\right]=4 \sum_{x \in \pi_{a b}} d(b, x)-4 \sum_{x \in \pi_{c b}} d(b, x) .
$$

Since $S S_{\pi}(a)<S S_{\pi}(b)$, then (10) implies

$$
\begin{aligned}
2 \sum_{x \in \pi_{a b}} d(b, x)-2 \sum_{x \in \pi_{a b c}} d(b, x)-2 \sum_{x \in \pi_{c b}} d(b, x)-|\pi|>0 . \\
2 \sum_{x \in \pi_{a b}} d(b, x)-2 \sum_{x \in \pi_{c b}} d(b, x)>2 \sum_{x \in \pi_{a b c}} d(b, x)+|\pi| .
\end{aligned}
$$

We use this to rewrite (11) in the following way

$$
\left[S S_{\pi}(b)-S S_{\pi}(a)\right]+\left[S S_{\pi}(c)-S S_{\pi}(b)\right]>4 \sum_{x \in \pi_{a b c}} d(b, x)+2|\pi| .
$$

From (5) we obtain

$$
\begin{aligned}
& S S_{\pi}(b)-S S_{\pi}(a)=2 R_{\pi}(b, a)+|\pi|, \\
& S S_{\pi}(c)-S S_{\pi}(b)=2 R_{\pi}(c, b)+|\pi| .
\end{aligned}
$$

Substituting this in (12), we get

$$
\left[2 R_{\pi}(b, a)+|\pi|\right]+\left[2 R_{\pi}(c, b)+|\pi|\right]>2 D_{\pi}(a, b, c)+2|\pi|,
$$

so, finally, we get

$$
R_{\pi}(b, a)+R_{\pi}(c, b)>D_{\pi}(a, b, c) .
$$

The following result is a simple corollary of Lemma 10.

Corollary 1 Let $\pi$ be a profile on $T$. If Mean $(\pi)=\{a\}$, then $R_{\pi}(b, a)+R_{\pi}(c, b)>$ $D_{\pi}(a, b, c)$ whenever $a b, b c \in E$.

Corollary 1 is the motivation for the definition of the following property for a location function. 


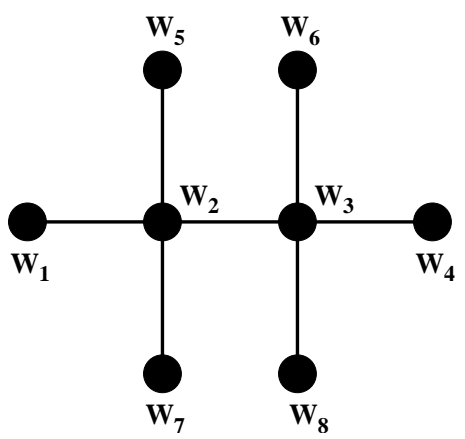

Figure 1: Mean does not satisfy converse of $(Z)$

Property (Z) : Let $\pi=\left(x_{1}, x_{2}, \ldots, x_{n}\right)$ be a profile and $L$ be a location function on $T$. If $L(\pi)=\{a\}$, then $R_{\pi}(b, a)+R_{\pi}(c, b)>D_{\pi}(a, b, c)$ whenever $a b, b c \in E$.

To get a feeling for this condition, note that the converse of property $(Z)$ does not necessarily hold when $L$ is the mean function for trees. We will give an example of a tree $T$ and a profile $\pi$ for which the converse of property $(Z)$ fails for the mean function.

Using the tree $T$ given in Fig. 1, define the profile $\pi=\left(w_{1}, w_{2}, w_{3}, w_{4}, w_{5}, w_{6}, w_{7}, w_{8}\right)$, and notice that $\operatorname{Mean}(\pi)=\left\{w_{2}, w_{3}\right\}$. For vertex $w_{2}$ we have

$$
\begin{aligned}
w_{2} w_{3}, w_{3} w_{4} & \in E, \\
R_{\pi}\left(w_{3}, w_{2}\right) & =-4, \\
R_{\pi}\left(w_{4}, w_{3}\right) & =8, \\
D_{\pi}\left(w_{2}, w_{3}, w_{4}\right) & =2,
\end{aligned}
$$

then

$$
R_{\pi}\left(w_{3}, w_{2}\right)+R_{\pi}\left(w_{4}, w_{3}\right)>D_{\pi}\left(w_{2}, w_{3}, w_{4}\right) .
$$

Notice that

$$
R_{\pi}\left(w_{4}, w_{3}\right)=R_{\pi}\left(w_{6}, w_{3}\right)=R_{\pi}\left(w_{8}, w_{3}\right)=7,
$$

this implies

$$
\begin{aligned}
& R_{\pi}\left(w_{3}, w_{2}\right)+R_{\pi}\left(w_{6}, w_{3}\right)>D_{\pi}\left(w_{2}, w_{3}, w_{6}\right), \\
& R_{\pi}\left(w_{3}, w_{2}\right)+R_{\pi}\left(w_{8}, w_{3}\right)>D_{\pi}\left(w_{2}, w_{3}, w_{8}\right) .
\end{aligned}
$$

From this we have that $w_{2}$ satisfies the condition:

$$
R_{\pi}\left(a, w_{2}\right)+R_{\pi}(b, a)>D_{\pi}\left(w_{2}, a, b\right) \text { whenever } w_{2} a, a b \in E .
$$

If we assume that the converse of property $(Z)$ is true, then $\operatorname{Mean}(\pi)=\left\{w_{2}\right\}$, and this contradicts that $\operatorname{Mean}(\pi)=\left\{w_{2}, w_{3}\right\}$.

Notice that a location function $L$ defined on the tree $\left(K_{2}\right)$ cannot get any additional features from property $(Z)$ because this property involves three distinct vertices. The next result shows that if $L$ satisfies axioms (Mid), $(C)$, and $(A)$, then it should be the mean function on $K_{2}$. 
Lemma 11 Let $T$ be a tree, and assume $a b \in E$. Let $\pi$ be a profile containing only vertices in the set $\{a, b\}$. If $L$ is a location function that satisfies axioms $(M i d),(C)$, and $(A)$, then $L(\pi)=\operatorname{Mean}(\pi)$.

Proof. By Lemma 1, $L$ also satisfies axiom $(F)$. Let $\pi$ be a profile containing only vertices in the set $\{a, b\}$. If $\pi$ contains an equal number of vertices $a$ and $b$, then we use the elements of $\pi$ to define the profile

$$
\alpha=\alpha_{1} \alpha_{2} \ldots \alpha_{n}
$$

where $\alpha_{i}=(a, b)$. Since $L$ satisfies $(C)$ and $(M i d)$, then

$$
L(\alpha)=L\left(\alpha_{1}\right) \cap L\left(\alpha_{2}\right) \cap \ldots \cap L\left(\alpha_{n}\right)=\{a, b\}
$$

and we also have

$$
\operatorname{Mean}(\alpha)=\operatorname{Mean}\left(\alpha_{1}\right) \cap \operatorname{Mean}\left(\alpha_{2}\right) \cap \ldots \cap \operatorname{Mean}\left(\alpha_{n}\right)=\{a, b\} .
$$

By axiom $(A)$ we obtain $L(\alpha)=L(\pi)=\operatorname{Mean}(\pi)$. On the other hand, if $\pi$ contains more vertices $a$ that vertices $b$, we define the profile

$$
\alpha=\alpha_{1} \alpha_{2} \ldots \alpha_{n} \beta
$$

where $\alpha_{i}=(a, b)$ and $\beta=(a, a, \ldots, a)$. From axioms $(C),(M i d)$, and $(F)$ we have

$$
L(\alpha)=L\left(\alpha_{1}\right) \cap L\left(\alpha_{2}\right) \cap \ldots L\left(\alpha_{n}\right) \cap L(\beta)=\{a\}
$$

and

$$
\operatorname{Mean}(\alpha)=\operatorname{Mean}\left(\alpha_{1}\right) \cap \operatorname{Mean}\left(\alpha_{2}\right) \cap \ldots \operatorname{Mean}\left(\alpha_{n}\right) \cap \operatorname{Mean}(\beta)=\{a\} .
$$

We use now axiom $(A)$ to conclude $L(\alpha)=L(\pi)=\operatorname{Mean}(\pi)$. Similarly, we can show that if $\pi$ contains more vertices $b$ that vertices $a$, then $L(\pi)=\operatorname{Mean}(\pi)$.

The next objective is to show that if $|L(\pi)|=|\operatorname{Mean}(\pi)|=1$ for some profile $\pi$, then $L(\pi)=\operatorname{Mean}(\pi)$ whenever $L$ satisfies axioms $(C),(M i d),(A)$, and property $(Z)$. In proving this result, we need to consider the cases when the vertex in Mean $(\pi)$ is a leaf or is not a leaf of $T$. First we prove that if $\operatorname{Mean}(\pi)=\{x\}$ and $L(\pi)=\{y\}$, then $x$ and $y$ are close to each other in $T$. Property $(Z)$ is the main ingredient in the proof of this result.

Lemma 12 Let $L$ be a location function on $T$ that satisfies axioms (Mid), $(A)$, and $(C)$. Let $\pi=\left(x_{1}, x_{2}, \ldots, x_{n}\right)$ be a profile on $T$. If $\operatorname{Mean}(\pi)=\{x\}, L(\pi)=\{y\}$, and $x \neq y$, then $d(x, y) \leq 1$. 
Proof. Assume $d(x, y)>1$, and let $P(x, y)=x v_{1} v_{2} \ldots v_{s-1} v_{s} y$ be the path from $x$ to $y$. By Lemma 3 we have that

$$
S S_{\pi}\left(v_{s-1}\right)<S S_{\pi}\left(v_{s}\right)<S S_{\pi}(y),
$$

Since $L$ satisfies axiom $(Z)$, we have

$$
R_{\pi}\left(v_{s}, y\right)+R_{\pi}\left(v_{s-1}, v_{s}\right)>D_{\pi}\left(y, v_{s-1}, v_{s}\right) .
$$

From (5) we obtain

$$
\begin{aligned}
R_{\pi}\left(v_{s}, y\right) & =\frac{S S_{\pi}\left(v_{s}\right)-S S_{\pi}(y)-|\pi|}{2}, \\
R_{\pi}\left(v_{s-1}, v_{s}\right) & =\frac{S S_{\pi}\left(v_{s-1}\right)-S S_{\pi}\left(v_{s}\right)-|\pi|}{2} .
\end{aligned}
$$

If we substitute this in (14) and simplify we get

$$
S S_{\pi}\left(v_{s-1}\right)-S S_{\pi}(y)>2 D_{\pi}\left(y, v_{s-1}, v_{s}\right)+2|\pi|>0 .
$$

This implies that $S S_{\pi}(y)<S S_{\pi}\left(v_{s-1}\right)$, and this contradicts (13). So we have $d(x, y) \leq$ 1.

From the previous result it follows that if we would have $\operatorname{Mean}(\pi)=\{v\}, L(\pi)=$ $\{u\}$, and $u \neq v$, then $u$ would be adjacent to $v$. Now, we prove that if $v$ is not a leaf, then $u=v$.

Lemma 13 Let $L$ be a location function on $T$ that satisfies axioms (Mid), $(A),(C)$, and property $(Z)$, and let $\pi=\left(x_{1}, x_{2}, \ldots, x_{n}\right)$ be a profile on $T$. If $v$ is not leaf, $|L(\pi)|=1$, and $\operatorname{Mean}(\pi)=\{v\}$, then $L(\pi)=\{v\}$.

Proof. Assume $L(\pi)=\{z\}$ and $v \neq z$, then Lemma 12 implies $d(v, z) \leq 1$. If $d(v, z)=1$, then there is a vertex $u$ such that $u v, v z \in E$. By Lemma 8 we have

$$
R_{\pi}(u, v)+R_{\pi}(z, v)=D_{\pi}(u, v, z) .
$$

Now $L$ satisfies axiom $(Z)$, hence

$$
R_{\pi}(v, u)+R_{\pi}(z, v)>D_{\pi}(u, v, z) .
$$

Subtracting (16) from (15), we obtain

$$
\begin{aligned}
R_{\pi}(u, v)-R_{\pi}(v, u) & <0 \\
R_{\pi}(u, v)-\left(-R_{\pi}(u, v)-|\pi|\right) & <0 \\
2 R_{\pi}(u, v)+|\pi| & <0 \\
S S_{\pi}(u)-S S_{\pi}(v) & <0 \quad \text { by }(6)
\end{aligned}
$$


This means that $S S_{\pi}(u)<S S_{\pi}(v)$, which contradicts that Mean $(\pi)=\{v\}$. Therefore, we conclude that $L(\pi)=\{v\}$.

We would like to extend this result to the case when $v$ is a leaf, but this requires us to prove some preparatory results. The next lemma shows that if the mean of a profile $\pi$ is a vertex that is a leaf, then this vertex is included in $\pi$.

Lemma 14 Let $\pi=\left(x_{1}, x_{2}, \ldots, x_{n}\right)$ be a profile on $T$. Let $v$ be a vertex that is a leaf and assume $\operatorname{Mean}(\pi)=\{v\}$, then $v$ is an element of $\pi$.

Proof. If $v$ is not an element of $\pi$, let $w$ be the vertex adjacent to $v$. Notice that

$$
d\left(v, x_{i}\right)=d\left(w, x_{i}\right)-1 \quad \forall i \in\{1,2, \ldots n\} .
$$

This implies $S S_{\pi}(w)<S S_{\pi}(v)$ which contradicts that Mean $(\pi)=\{v\}$. Hence, $v$ is included in $\pi$.

Next we characterize the profiles $\pi$ having the property that their mean contains two vertices, and one of these vertices is a leaf.

Lemma 15 Let $\pi=\left(x_{1}, x_{2}, \ldots, x_{n}\right)$ be a profile on $T$. If $\operatorname{Mean}(\pi)=\{v, x\}$ and $v$ is a leaf of $T$, then the only vertices in $\pi$ are $x$ and $v$. Moreover, $\pi$ contains an equal number of vertices $x$ and $v$.

Proof. If $w$ is a vertex that belongs to $\pi$ and is different from $x$ and $v$, then $d(v, w)=$ $d(x, w)+1$. This implies that $S S_{\pi}(v)<S S_{\pi}(x)$, which contradicts Mean $(\pi)=\{v, x\}$. Consequently, $\pi$ cannot contain any vertex different from $x$ and $v$. Notice that if $\pi$ does not contain an equal number of $x$ and $v$, then $|\operatorname{Mean}(\pi)|=1$, which contradicts $|\operatorname{Mean}(\pi)|=2$.

If $L$ is a location function that satisfies axioms $(M i d),(A)$, and $(C)$, then Lemmas 11 and 15 provide the following corollary that characterizes the set $L(\pi)$ when this set contains two elements and one of them is a leaf.

Corollary 2 Let $L$ be a location function on $T$ that satisfies axioms (Mid), $(A)$, and $(C)$. Let $\pi=\left(x_{1}, x_{2}, \ldots, x_{n}\right)$ be a profile on $T$ and assume $v$ is a leaf. If Mean $(\pi)=$ $\{v, x\}$, then $L(\pi)=\{v, x\}$.

The next result tells us that the sets $\operatorname{Mean}(\pi)$ and $L(\pi)$ are equal when the set $\operatorname{Mean}(\pi)$ contains a leaf of $T$.

Lemma 16 Let $L$ be a location function on $T$ that satisfies axioms (Mid), $(A)$, and $(C)$, and let $\pi=\left(x_{1}, x_{2}, \ldots, x_{n}\right)$ be a profile on $T$. If $y$ is a leaf of $T$ such that $y \in \operatorname{Mean}(\pi)$, then $\operatorname{Mean}(\pi)=L(\pi)$. 
Proof. To prove the result, we use induction on $|\pi|$. If $|\pi|=1$, then $\pi=(y)$, and in this case $\operatorname{Mean}(\pi)=\{y\}$ and, by Faithfulness, we have $L(\pi)=\{y\}$. Assume the result is true for any profile with at most $n$ vertices, and let $\pi$ be a profile with $n+1$ vertices such that $y \in \operatorname{Mean}(\pi)$ and $y$ is a leaf. Assume Mean $(\pi)=\{y\}$ and denote by $\alpha$ the profile that contains all the vertices of $\pi$ except for one occurrence of $y$. Since Mean $(\alpha \diamond y)=\{y\}$, Lemma 7 implies that $y \in M e a n(\alpha)$. From Lemma 14 it follows that $y$ occurs in $\alpha$. Since $\alpha$ contains $n$ vertices, the induction hypothesis yields $\operatorname{Mean}(\alpha)=L(\alpha)$. Now we use Lemma 6 to conclude that $L(\pi)=L(\alpha \diamond y)=\{y\}=$ $\operatorname{Mean}(\pi)$. On the other hand, it follows from Corollary 2 that if $\operatorname{Mean}(\pi)=\{y, x\}$, then $L(\pi)=\{y, x\}$.

We are now in position to show that the sets $\operatorname{Mean}(\pi)$ and $L(\pi)$ are the same when the mean of $\pi$ is a leaf, $L$ satisfies property $(Z)$, and $|\operatorname{Mean}(\pi)|=|L(\pi)|=1$.

Lemma 17 Let $L$ be a location function on $T$ that satisfies axioms $(M i d),(A),(C)$, and property $(Z)$, and let $\pi=\left(x_{1}, x_{2}, \ldots, x_{n}\right)$ be a profile on $T$. If $y$ is a leaf and $|\operatorname{Mean}(\pi)|=|L(\pi)|=1$, then Mean $(\pi)=\{y\}$ if and only if $L(\pi)=\{y\}$.

Proof. By Lemma 16, we know that $\operatorname{Mean}(\pi)=\{y\}$ and implies $L(\pi)=\{y\}$.

On the other hand, assume $L(\pi)=\{y\}$ and $|\operatorname{Mean}(\pi)|=1$. If $\operatorname{Mean}(\pi)=\{z\}$ and $z$ is a leaf, then Lemma 16 implies $z=y$. If $z$ is not a leaf then Lemma 13 implies that $z=y$. Therefore we have $\operatorname{Mean}(\pi)=\{y\}$.

A consequence of Lemmas 13 and 17 is the following generalization of Lemma 17.

Lemma 18 Let $L$ be a location function on $T$ that satisfies axioms $(M i d),(A),(C)$, and property $(Z)$, and let $\pi=\left(x_{1}, x_{2}, \ldots, x_{n}\right)$ be a profile on $T$. If $\mid$ Mean $(\pi) \mid=$ $|L(\pi)|=1$, then Mean $(\pi)=\{y\}$ if and only if $L(\pi)=\{y\}$.

Finally we prove that if $\pi$ is a profile having an even number of vertices, and $L$ is a location function that satisfies axioms $(M i d),(C),(A)$, and property $(Z)$, then the sets $L(\pi)$ and $\operatorname{Mean}(\pi)$ are equal.

Lemma 19 Let $\pi=\left(x_{1}, x_{2}, \ldots, x_{n}\right)$ be a profile on $T$, and let $L$ be a location function that satisfies axioms $(M i d),(C),(A)$, and property $(Z)$. If $|\pi|$ is even, then $L(\pi)=$ $\operatorname{Mean}(\pi)$.

Proof. Note that if all the elements of $\pi$ belong to the set $\{a, b\}$ and $a b \in E$, then Lemma 11 implies that $L(\pi)=\operatorname{Mean}(\pi)$. Now, assume $\pi$ contains at least three distinct vertices and also assume $L(\pi)=\left\{a_{1}, a_{2}, \ldots, a_{k}\right\}$. By Lemma 6 , we get

$$
\begin{aligned}
L\left(\pi \diamond a_{1}\right) & =\left\{a_{1}\right\}, \\
L\left(\pi \diamond a_{2}\right) & =\left\{a_{2}\right\}, \\
\vdots & \\
L\left(\pi \diamond a_{s}\right) & =\left\{a_{k}\right\} .
\end{aligned}
$$


Since $|\pi|$ is even, $\left|\pi \diamond a_{i}\right|$ is odd, and, by Lemma 9, we have

$$
\left|\operatorname{Mean}\left(\pi \diamond a_{i}\right)\right|=1 \quad \forall 1 \leq i \leq k .
$$

From Lemma 18 we get

$$
L\left(\pi \diamond a_{i}\right)=\operatorname{Mean}\left(\pi \diamond a_{i}\right) \quad \forall 1 \leq i \leq k .
$$

Observe that (17) and Lemma 7 yield $\left\{a_{1}, a_{2}, \ldots, a_{k}\right\} \subseteq$ Mean $(\pi)$. This implies that $k \leq 2$, since $|\operatorname{Mean}(\pi)| \leq 2$. If $L(\pi)=\left\{a_{1}, a_{2}\right\}$, we have $L(\pi)=\operatorname{Mean}(\pi)=\left\{a_{1}, a_{2}\right\}$. On the other hand, if $|L(\pi)|=|\operatorname{Mean}(\pi)|=1$, then, by Lemma 18, we get $L(\pi)=$ $\operatorname{Mean}(\pi)$.

To finish the proof we just need to show that $|L(\pi)|=1$ forces $|\operatorname{Mean}(\pi)|=1$. So assume $L(\pi)=\{c\}$ and $\mid$ Mean $(\pi) \mid=2$. By Lemma 6 we have $L(\pi \diamond c)=\{c\}$, and, since $|\pi \diamond c|$ is odd, we have $\mid$ Mean $(\pi \diamond c) \mid=1$. Lemma 18 implies that Mean $(\pi \diamond c)=L(\pi \diamond c)=$ $\{c\}$, and Lemma 7 shows that $c \in \operatorname{Mean}(\pi)$. This means that $\operatorname{Mean}(\pi)=\{b, c\}$, for some vertex $b$ distinct from $c$, and also that $b$ and $c$ are adjacent. First assume that $b$ is a leaf of $T$. Then, by Corollary 2 , we have $L(\pi)=\{c, b\}$, which contradicts $L(\pi)=\{c\}$. So $b$ cannot be a leaf of $T$, and we can find a vertex $z$ such that $c b, b z \in E$. Because $L$ satisfies property $(Z)$, we get

$$
R_{\pi}(b, c)+R_{\pi}(z, b)>D_{\pi}(c, b, z) .
$$

Moreover, Lemma 8 shows

$$
R_{\pi}(c, b)+R_{\pi}(z, b)=D_{\pi}(c, b, z) .
$$

If we substitute this in (18) and simplify, we get

$$
R_{\pi}(b, c)>R_{\pi}(c, b)
$$

On the other hand, since $\operatorname{Mean}(\pi)=\{c, b\}$, we have $S S_{\pi}(c)=S S_{\pi}(b)$. From (5) we obtain

$$
\begin{aligned}
S S_{\pi}(c)-S S_{\pi}(b) & =S S_{\pi}(b)-S S_{\pi}(c) \\
2 R_{\pi}(c, b)+|\pi| & =2 R_{\pi}(b, c)+|\pi| \\
R_{\pi}(c, b) & =R_{\pi}(b, c) .
\end{aligned}
$$

It is clear that (20) contradicts (19). This final contradiction implies that $\mid$ Mean $(\pi) \mid=$ 2 is not possible. Therefore it follows that if $|L(\pi)|=1$, then $|\operatorname{Mean}(\pi)|=1$, which concludes the proof.

\section{The Main Result and Independence of Axioms}

Finally, we put the above results together in the proof of our main result. 
Theorem 1 Let $L$ be a location function on a finite tree $T$. Then $L$ is the mean function if and only if $L$ satisfies axioms $(A),(M i d),(C)$, and property $(Z)$.

Proof. It is immediate that if $L$ is the mean function, then $L$ satisfies axioms $(A)$, $(M i d),(C)$, and property $(Z)$. On the other hand, assume that $L$ satisfies axioms $(A)$, $(M i d),(C)$, and property $(Z)$. By Lemma 19 , we have $L(\pi)=\operatorname{Mean}(\pi)$ whenever $|\pi|$ is even. In the case that $|\pi|$ is odd we have $|\operatorname{Mean}(\pi)|=1$, by Lemma 9. Hence, by Lemma 18, we have $L(\pi)=\operatorname{Mean}(\pi)$, and we are done.

Next, we establish the independence of the axioms $(A),(M i d),(C)$, and property $(Z)$.

- (Mid), (A), and (C) are independent from property $(\mathbf{Z})$.

The cube function satisfies axioms $(M i d),(A)$, and $(C)$. Consider the path $P\left(v_{1}, v_{10}\right)=v_{1} v_{2} \ldots v_{10}$ and the profile $\pi=\left(v_{1}, v_{2}, v_{10}\right)$. It is not difficult to verify that $\operatorname{Mean}(\pi)=\left\{v_{4}\right\}$ and $C u b e(\pi)=\left\{v_{5}\right\}$. Notice that $v_{5} v_{4}$ and $v_{4} v_{3}$ are edges of $P\left(v_{1}, v_{10}\right)$ and that $D_{\pi}\left(v_{5}, v_{4}, v_{3}\right)=0$. Then we have

$$
R_{\pi}\left(v_{4}, v_{5}\right)+R_{\pi}\left(v_{3}, v_{4}\right)=-2+1=-1<D_{\pi}\left(v_{5}, v_{4}, v_{3}\right) .
$$

Since $R_{\pi}\left(v_{4}, v_{5}\right)+R_{\pi}\left(v_{3}, v_{4}\right)<D_{\pi}\left(v_{5}, v_{4}, v_{3}\right)$, the cube function does not satisfies property $(Z)$.

- (C), (A), and property (Z) are independent from (Mid). Consider the path $P\left(v_{0}, v_{3}\right)=v_{0} v_{1} v_{2} v_{3}$, and let $\pi$ be a profile on $P\left(v_{0}, v_{3}\right)$. Define the following location function

$$
L(\pi)= \begin{cases}\operatorname{Mean}(\pi) & \text { if }|\operatorname{Mean}(\pi)|=1 . \\ \left\{v_{1}\right\} & \text { if } \operatorname{Mean}(\pi)=\left\{v_{0}, v_{1}\right\} . \\ \left\{v_{2}\right\} & \text { if } \operatorname{Mean}(\pi)=\left\{v_{2}, v_{3}\right\} . \\ \left\{v_{1}, v_{2}\right\} & \text { if } \operatorname{Mean}(\pi)=\left\{v_{1}, v_{2}\right\} .\end{cases}
$$

Because the mean function satisfies axioms $(A)$ and $(C)$, function $L$ satisfies these axioms too. Notice also that $L$ satisfies property $Z$ for profiles $\pi$ for which $|\operatorname{Mean}(\pi)|=1$. If $\operatorname{Mean}(\pi)=\left\{v_{0}, v_{1}\right\}$, then the definition of $L$ indicates $L(\pi)=$ $\left\{v_{1}\right\}$. We need to verify property $(Z)$ in this case. Mean $(\pi)=\left\{v_{0}, v_{1}\right\}$ implies $\pi$ contains an equal number $k$ of vertices $v_{0}$ and $v_{1}$. We can see that $v_{2}$ and $v_{3}$ are the unique vertices satisfying $v_{1} v_{2}, v_{2} v_{3} \in E\left(P\left(v_{0}, v_{3}\right)\right)$. Furthermore,

$$
R_{\pi}\left(v_{2}, v_{1}\right)+R_{\pi}\left(v_{3}, v_{2}\right)=k+3 k>D_{\pi}\left(v_{1}, v_{2}, v_{3}\right)=0
$$

then $L$ satisfies property $(Z)$ for this $\pi$.

A similar argument can be used to prove that $L$ satisfies property $(Z)$ for profiles $\pi$ with $\operatorname{Mean}(\pi)=\left\{v_{2}, v_{3}\right\}$. This establishes that $L$ satisfies $(A),(C)$, and property $(Z)$; however, it does not satisfies $(M i d)$ because $L\left(\left(v_{0}, v_{1}\right)\right) \neq\left\{v_{0}, v_{1}\right\}$. 
- (Mid), (A), and property $(\mathbf{Z})$ are independent from $(\mathbf{C})$. We will use the tree $T$ defined in Fig. 1 above. Denote by $\beta$ the profile on $T$ having each vertex of $T$ an equal number of times. Let $\pi$ be a profile on $T$ and define the following location function

$$
L(\pi)= \begin{cases}\left\{w_{2}\right\} & \text { if } \pi \text { contain every vertex of } T \text { an equal number of times. } \\ \operatorname{Mean}(\pi) & \text { otherwise. }\end{cases}
$$

We have determined above that $w_{2}$ satisfies the condition

$$
R_{\pi}\left(b, w_{2}\right)+R_{\pi}(c, b)>D_{\pi}\left(w_{2}, b, c\right) \text { whenever } w_{2} b, b c \in E .
$$

Consequently, $L$ satisfies property $(Z)$ if $\pi$ is a profile containing every vertex of $T$ an equal number of times. $L$ satisfies property $(Z)$ for any other profile because the mean function does satisfies this axiom. From the definition of $L$ we obtain

$$
\begin{aligned}
& \operatorname{Mean}\left(\left(w_{1}, w_{4}\right)\right)=L\left(\left(w_{1}, w_{4}\right)\right)=\left\{w_{2}, w_{3}\right\}, \\
& \operatorname{Mean}\left(\left(w_{5}, w_{8}\right)\right)=L\left(\left(w_{5}, w_{8}\right)\right)=\left\{w_{2}, w_{3}\right\}, \\
& \operatorname{Mean}\left(\left(w_{7}, w_{6}\right)\right)=L\left(\left(w_{7}, w_{6}\right)\right)=\left\{w_{2}, w_{3}\right\}, \\
& \operatorname{Mean}\left(\left(w_{2}, w_{3}\right)\right)=L\left(\left(w_{2}, w_{3}\right)\right)=\left\{w_{2}, w_{3}\right\} .
\end{aligned}
$$

Assume $L$ satisfies axiom $(C)$, then

$$
\begin{aligned}
L\left(\left(w_{1}, w_{4}, w_{2}, w_{3}\right)\right) & =L\left(\left(w_{1}, w_{4}\right)\right) \cap L\left(\left(w_{2}, w_{3}\right)\right) \\
L\left(\left(w_{1}, w_{4}, w_{2}, w_{3}, w_{7}, w_{6}\right)\right) & =L\left(\left(w_{1}, w_{4}, w_{2}, w_{3}\right)\right) \cap L\left(\left(w_{7}, w_{6}\right)\right), \\
L\left(\left(w_{1}, w_{4}, w_{2}, w_{3}, w_{7}, w_{6}, w_{5}, w_{8}\right)\right) & =L\left(\left(w_{1}, w_{4}, w_{2}, w_{3}, w_{7}, w_{6}\right)\right) \cap L\left(\left(w_{5}, w_{8}\right)\right) .
\end{aligned}
$$

Note that all these sets are equal to $\left\{w_{2}, w_{3}\right\}$. Nevertheless, according to the definition of $L$ we have

$$
L\left(\left(w_{1}, w_{4}, w_{2}, w_{3}, w_{7}, w_{6}, w_{5}, w_{8}\right)\right)=\left\{w_{2}\right\} .
$$

From this we conclude that $L$ does not satisfies $(C)$.

- (C), (Mid), and property (Z) are independent from (A).

Consider the tree $T=a b$ with only two vertices. Let $\pi$ be a profile on $T$. We say that $\pi$ satisfies condition $(W)$ if

(i) $|\pi|>2$,

(ii) $\pi$ contains an equal number of $a^{\prime} s$ and $b^{\prime} s$,

(iii) if $\pi=\beta_{1} \beta_{2} \ldots \beta_{k}$, then $L\left(\beta_{1}\right) \cap L\left(\beta_{2}\right) \cap \cdots \cap L\left(\beta_{k}\right)=\emptyset$.

An example of a profile that satisfies condition $(W)$ is $\pi=(a, a, b, b)$. Define the following location function

$$
L(\pi)= \begin{cases}\operatorname{Mean}(\pi) & \text { if } \pi \text { contains more } a^{\prime} s \text { than } b^{\prime} s \text { or more } b^{\prime} s \text { than } a^{\prime} s . \\ \{a, b\} & \text { if } \pi=(a, b) \\ \{a\} & \text { if } \pi \text { satisfies condition } W .\end{cases}
$$


We see that $L$ satisfies axioms $(C),(M i d)$, and property $(Z)$. It does not satisfies $(A)$ and it is not equal to the mean function because $L((a, a, b, b))=\{a\} \neq\{a, b\}=\operatorname{Mean}((a, a, b, b))$.

\section{References}

[1] K.J. Arrow, A.K. Sen, K. Suzumura (eds), Handbook of social choice and welfare, Volumes 1, North Holland, Amsterdam, 2002.

[2] K.J. Arrow, A.K. Sen, K. Suzumura (eds), Handbook of social choice and welfare, Volumes 2, North Holland, Amsterdam, 2005.

[3] J.P. Barthélemy, M.F. Janowitz, A formal theory of consensus, SIAM J. Discr. Math. 4 (1991) $305-322$.

[4] J.P. Barthélemy, B. Monjardet, The median procedure in cluster analysis and social choice theory, Math. Soc. Sci. 1 (1981) 235 - 268.

[5] J.E. Biagi, Facility location and the mean function, MA Thesis, University of Louisville, 2000.

[6] W.H.E. Day, F.R. McMorris, Axiomatic consensus theory in group choice and biomathematics, Frontiers in Appl. Math., SIAM, Philadelphia, 2003.

[7] D.P. Foster, R. Vohra, An axiomatic characterization of a class of location in tree networks, Operational Research 46 (1998) 347 - 354.

[8] R. Holzman, An axiomatic approach to location on networks, Mathematics of Operations Research 15 (1990) $553-563$.

[9] F.R. McMorris, H.M. Mulder, R.C. Powers, The median function on distributive semilattices, Discrete Applied Math. 127 (2003) 319 - 324.

[10] F.R. McMorris, H.M. Mulder, F.S. Roberts, The median procedure on median graphs, Discr. Appl. Math. 84 (1998) $165-181$.

[11] F.R. McMorris, F.S. Roberts, C. Wang, The center function on trees, Networks 38 (2001) $84-87$.

[12] P.B. Mirchandani and R.L. Francis (eds), Discrete location theory, Wiley, New York, 1990.

[13] H.M. Mulder, K.B. Reid, M. Pelsmajer, Axiomization of the center function on trees, Australasian J. Combinatorics 41 (2008) 223 - 226.

[14] R. Vohra, An axiomatic characterization of some location in trees, European Journal of Operational Research 90 (1996) $78-84$. 